\title{
On the Dimer Problem and the Ising Problem in Finite 3-dimensional Lattices
}

\author{
Martin Loebl * \\ Department of Applied Mathematics \\ and \\ Institute for Theoretical Computer Science (ITI) \\ Charles University \\ Malostranske n. 25, 11800 Praha 1, Czech Republic \\ loebl@kam-enterprize.ms.mff.cuni.cz \\ Submitted: April 11, 2001; Accepted: July 8, 2002 \\ MR Subject Classifications: 05B35, 05C15, 05A15
}

\begin{abstract}
We present a new expression for the partition function of the dimer arrangements and the Ising partition function of the 3-dimensional cubic lattice. We use the Pfaffian method. The partition functions are expressed by means of expectations of determinants and Pfaffians of matrices associated with the cubic lattice.
\end{abstract}

\section{Introduction}

The close-packed dimer model of statistical mechanics can be stated as follows. One considers a set of sites and a set of bonds connecting certain pairs of sites. Each bond $b$ can absorb a 'dimer' (which represents a diatomic molecule) with corresponding energy $E_{b}$. It is required that each site is occupied exactly once by one of the atoms of a dimer. A state $s$ is an arrangement of dimers which meets this requirement, and its energy $E(s)$ is $\sum E_{b}$ where the sum is taken over all bonds $b$ which absorb a dimer. Then the partition function of the dimer model may be viewed as a density function of energy levels.

The dimer model was first considered by Roberts [18] in 1935, and by Fowler and Rushbrook [3]. The dimer model for 2-dimensional lattices appears in calculations of the thermodynamic properties of a system of diatomic molecules-dimers. It has been solved

*Partially supported by the Project LN00A056 of the Czech Ministry of Education, by GAUK 158 grant, and by FONDAP on applied mathematics 
by Kasteleyn [13] and by Temperley and Fisher [11]. The same problem for 3-dimensional lattices remains an important open problem of statistical physics (see [14] for references).

Many fundamental observations about the dimer and monomer-dimer model in general lattice graphs have been given by Heilmann and Lieb [9], [10].

Another model we consider here is the Ising version of the Edwards-Anderson model. It can be described as follows. A coupling constant $J_{i j}$ is assigned to each bond $\{i, j\}$ of a given lattice graph G; the coupling constant characterizes the interaction between the particles represented by sites $i$ and $j$. A physical state of the system is an assignment of spin $\sigma_{i} \in\{+1,-1\}$ to each site $i$. The Hamiltonian (or energy function) is defined as $H(\sigma)=-\sum_{\{i, j\} \in E} J_{i j} \sigma_{i} \sigma_{j}$. The distribution of physical states over all possible energy levels is encapsulated in the partition function $Z(\beta)=\sum_{\sigma} e^{-\beta H(\sigma)}$ from which all fundamental physical quantities may be derived.

The literature on the 3-dimensional dimer problem and the 3-dimensional Ising problem is vast but there is a general feeling and some evidence (see e.g. [12]) that no closed solution similar to the solutions of the 2-dimensional case nor a deterministic efficient algorithm may be found for the cubic lattices.

This however does not rule out a statistical treatment. We believe that our new expressions are natural enough to allow such further analysis.

Recent papers [16], [17] also study the problems using a Pfaffian method. They obtain new expressions by means of a series of Pfaffians with a topological signature. Our approach is more combinatorial in nature. We express the partition functions by means of expectations of the determinants of matrices naturally associated with the cubic lattice. Determinants and spectral properties of random matrices are extensively studied (see e.g. [8]) and a goal of this paper is to draw attention to possible applications of related machinery to the 3 -dimensional statistical mechanics problems.

We may reformulate the dimer problem and the Ising problem in graph theoretic terms as follows. A graph is a pair $G=(V, E)$ where $V$ is a set of vertices and $E$ is now the set of edges (not the energy). A graph with some regularity properties may be called a lattice graph. We associate with each edge $e$ of $G$ a weight $w(e)$ and for a subset of edges $A \subset E, w(A)$ will denote the sum of the weights $w(e)$ associated with the edges in $A$.

A subset of edges $P \subset E$ is called a perfect matching or dimer arrangement if each vertex belongs to exactly one element of $P$. The dimer partition function may be viewed as a polynomial $\mathcal{P}(G, x)$ which equals the sum of $x^{w(P)}$ over all perfect matchings $P$ of $G$. This polynomial is also called the generating function of perfect matchings.

The Ising partition function is very close to the generating function of cuts which is a standard concept in graph theory. A cut of a graph $G=(V, E)$ is a partition of its vertices into two disjoint subsets $V_{1}, V_{2} \subset V$, and the implied set of edges between the two parts:

$$
C\left(V_{1}, V_{2}\right)=\left\{\{u, v\} \in E: u \in V_{1}, v \in V_{2}\right\}
$$

The generating function of cuts $\mathcal{C}(G, x)$ equals the sum of $x^{w(C)}$ over all cuts $C$ of $G$.

If we set the coupling constant $J_{i j}$ as the weight $w(\{i, j\})$ of the edge $\{i, j\}$, the 
generating function of cuts becomes very similar to the partition function:

$$
Z(\beta)=2 \sum_{\text {cutC }} e^{-\beta(2 w(C)-W)}=2 e^{\beta W} \mathcal{C}\left(G, e^{-2 \beta}\right)
$$

where $W$ is the sum of all the edge weights.

The generating functions of perfect matchings and cuts may be defined in a more general way as follows: associate a variable $x_{e}$ with each edge $e$ of graph $G$, let $x(A)=$ $\prod_{e \in A} x_{e}$ and let e.g. the generating function of perfect matchings be the sum of $x(P), P$ perfect matching of $G$. All results introduced in this paper also hold in this more general setting; however the presentation using weights rather than variables is perhaps more natural.

This paper studies properties of finite cubic lattices. Let us now fix some notation for them. Let $m$ be an odd positive integer and $k$ an even positive integer. The cubic lattice $Q_{m m k}$ is the following graph:

$Q_{m m k}$ has vertices $V_{x y z}, x, y=1, \ldots, m, z=1, \ldots, k$, and the following edges:

1. The vertical edges $v_{x y z}=\left\{V_{x y z}, V_{x y(z+1)}\right\}$,

$$
z=1, \ldots, k-1 \text {, }
$$

2. The width edges $w_{x y z}=\left\{V_{x y z}, V_{x(y+1) z}\right\}$,

$$
y=1, \ldots, m-1
$$

3. The horizontal edges $h_{x y z}=\left\{V_{x y z}, V_{(x+1) y z}\right\}$,

$$
x=1, \ldots m-1 \text {. }
$$

Let us denote the ordered set $\left(V_{x y 1}, \ldots, V_{x y k}\right)$ by $V_{x y}$. $V_{x y}$ will also stand for the vertical path of $Q_{m m k}$ from $V_{x y 1}$ to $V_{x y k}$. Let $\bar{V}_{x y}$ denote the reversal of $V_{x y}$.

$Q_{m m k}$ is a bipartite graph, which means that its vertices may be partitioned into two sets $Z_{1}, Z_{2}$ such that if $e$ is an edge of $Q_{m m k}$ then $\left|e \cap Z_{1}\right|=\left|e \cap Z_{2}\right|=1$. Moreover, we have also that $\left|Z_{1}\right|=\left|Z_{2}\right|=m m k / 2$. Let $\mathcal{Z}$ be the square $\left(Z_{1} \times Z_{2}\right)$ matrix defined by $\mathcal{Z}_{i j}=x^{w(i j)}$ if $e=\{i j\}$ is an edge of $Q_{m m k}$ with weight $w(e)=w(i j)$, and $\mathcal{Z}_{i j}=0$ otherwise.

We will consider matrix $\mathcal{Z}$ with its rows and columns ordered in agreement with the natural order $\left(V_{11}, \bar{V}_{12}, \ldots, V_{1 m}, \bar{V}_{21}, \ldots, V_{m m}\right)$ and we will assume that $V_{111} \in Z_{1}$.

Note that $\mathcal{P}\left(Q_{m m k}, x\right)$ equals the permanent of $\mathcal{Z}$. In this paper we show that $\mathcal{P}\left(Q_{m m k}, x\right)$ may be computed from the average of determinants of CERTAIN signings of $\mathcal{Z}$, where a signing of a matrix is obtained by multiplying some of the entries of the matrix by -1 .

The signings of $\mathcal{Z}$ correspond to orientations of $Q_{m m k}$.

An orientation of a graph $G=(V, E)$ is a digraph $D=(V, A)$ obtained from $G$ by assigning an orientation to each edge of $G$, i.e., by ordering the elements of each edge of $G$. The elements of $A$ are called arcs. We say that signing $Z$ of $\mathcal{Z}$ corresponds to 
orientation $D$ of $Q_{m m k}$ if $Z_{i j}=x^{w(i j)}$ if $(i j) \in A(D), Z_{i j}=-x^{w(i j)}$ if $(j i) \in A(D)$, and $Z_{i j}=0$ otherwise.

An expression of similar flavor as our result exists already: a seminal observation of Heilmann and Lieb [9], [10] asserts that $\mathcal{P}\left(Q_{m m k}, x^{2}\right)$ equals the average of $(\operatorname{det}(Z))^{2}$ over ALL signings $Z$ of $\mathcal{Z}$.

The following short proof of this observation is taken from the monograph [15]. If $D$ is an orientation of $Q_{m m k}$ then let $A(D)$ denote the skew-symmetric adjacency matrix of $D$, i.e. matrix consisting of 4 blocks where both blocks on the main diagonal are 0-matrices and the remaining two blocks equal $Z$ and $-Z$, where $Z$ is the signing of $\mathcal{Z}$ corresponding to $D$. Clearly $\operatorname{det}(A(D))=(\operatorname{det}(Z))^{2}$, hence we need to show that $\mathcal{P}\left(Q_{m m k}, x^{2}\right)$ equals the expectation of $\operatorname{det}(A(D))$ over all orientations $D$ of $Q_{m m k}$. For the expectation we have

$$
E(\operatorname{det}(A(D)))=\sum \operatorname{sgn}(\pi) E\left(a_{1 \pi(1)} \ldots a_{n \pi(n)}\right)
$$

where $n=m m k$ and $A(D)=\left(a_{i j}\right)$ by the linearity of expectation. If $\pi$ is a permutation having a fix point or such that $i$ and $\pi(i)$ are non-adjacent for some $i \leq n$ then the term corresponding to $\pi$ equals 0 . If there is $i$ such that $\pi(\pi(i)) \neq i$ then the random variable $a_{i \pi(i)}$ occurs in the term corresponding to $\pi$ but the random variable $a_{\pi(i) i}$ does not. Hence

$$
E\left(a_{1 \pi(1)} \ldots a_{n \pi(n)}\right)=E\left(a_{i \pi(i)}\right) E\left(a_{1 \pi(1)} \ldots a_{(i-1) \pi(i-1)} a_{(i+1) \pi(i+1)} \ldots a_{n \pi(n)}\right)=0 .
$$

So we are left with the terms corresponding to those permutations which have no fix point, for which $i$ and $\pi(i)$ are adjacent and $(\pi)^{2}$ is the identity. Such permutations uniquely correspond to perfect matchings of $Q_{m m k}$ and the signs turn out correct.

A difference between our expression and the result of Heilmann and Lieb is that we replace the average of a multi quadratic function by the average of a multi linear function, with a restricted range. 


\subsection{Statement of the main result.}

An orientation $D$ of $Q_{m m k}$ is called stable if all vertical edges are oriented in $D$ from the 'smaller' to the 'bigger' vertex in the natural order. For edge e we let $s_{D}(e)=0$ if the orientation of e agrees with the natural order, and $s_{D}(e)=1$ otherwise.

\section{Theorem 1.1}

$$
\mathcal{P}\left(Q_{m m k}, x\right)=-2^{C_{r}} x^{w(M)}+\alpha\left(2^{C_{r}}+1\right)
$$

where $C_{r}=k m(m-1), M$ is the unique perfect matching of $Q_{m m k}$ consisting of vertical edges only and $\alpha$ equals the average of $\operatorname{det}(Z(D))$, D stable orientation of $Q_{m m k}$ satisfying

$$
\sum_{A} s_{D}\left(w_{x, 2 y, z}\right) s_{D}\left(w_{x, 2 y^{\prime}-1, z^{\prime}}\right)=\sum_{B} s_{D}\left(h_{2 x, y, z}\right) s_{D}\left(h_{2 x^{\prime}-1, y^{\prime}, z^{\prime}}\right)
$$

modulo 2, where

$A=\left\{\left(x, y, z, y^{\prime}, z^{\prime}\right) ; 1 \leq x \leq m, 1 \leq y \leq(m-1) / 2,1 \leq z \leq k, 1 \leq y^{\prime} \leq y, z \leq z^{\prime} \leq z+1\right\}$

and

$$
\begin{gathered}
B=\left\{\left(x, y, z, x^{\prime}, y^{\prime}, z^{\prime}\right) ; 1 \leq x \leq(m-1) / 2,1 \leq z \leq k, 1 \leq y \leq m, 1 \leq x^{\prime} \leq x,\right. \\
\left.(y, z) \leq\left(y^{\prime}, z^{\prime}\right) \leq\left(y^{\prime \prime}, z^{\prime \prime}\right)\right\} .
\end{gathered}
$$

In the definition of $B$ the order on pairs of integers is lexicographic order and $\left(y^{\prime \prime}, z^{\prime \prime}\right)$ is the immediate successor of $(y, z)$; if the immediate successor does not exist than we let $\left(y^{\prime \prime}, z^{\prime \prime}\right)=(y, z)$.

Theorem 1.1 holds also for $\mathcal{P}\left(Q_{m_{1} m_{2} k}, x\right)$ with $m_{1} \neq m_{2}$ odd. In this more general setting $C_{r}=1 / 2 k m_{1}\left(m_{2}-1\right)+1 / 2 k m_{2}\left(m_{1}-1\right)$,

$A=\left\{\left(x, y, z, y^{\prime}, z^{\prime}\right) ; 1 \leq x \leq m_{1}, 1 \leq y \leq\left(m_{2}-1\right) / 2,1 \leq z \leq k, 1 \leq y^{\prime} \leq y, z \leq z^{\prime} \leq z+1\right\}$

and

$$
\begin{gathered}
B=\left\{\left(x, y, z, x^{\prime}, y^{\prime}, z^{\prime}\right) ; 1 \leq x \leq\left(m_{1}-1\right) / 2,1 \leq z \leq k, 1 \leq y \leq m_{2}, 1 \leq x^{\prime} \leq x,\right. \\
\left.(y, z) \leq\left(y^{\prime}, z^{\prime}\right) \leq\left(y^{\prime \prime}, z^{\prime \prime}\right)\right\} .
\end{gathered}
$$

Example. Let us illustrate the statement of Theorem 1.1 by calculation of $\mathcal{P}\left(Q_{3,1,2}, x\right)$ with $w(e)=0$ for each edge e. $Q_{3,1,2}$ has no width edges: it is simply a square $(3 \times 2)$ grid and thus it has 6 vertices and 3 dimer arrangements. Hence $\mathcal{P}\left(Q_{3,1,2}, x\right)=3$. 
On the other hand there are $2^{4}$ stable orientations of $Q_{3,1,2}$ and those relevant for $\alpha$ are characterized by the equation

$$
s_{D}\left(h_{2,1,1}\right) s_{D}\left(h_{1,1,1}\right)+s_{D}\left(h_{2,1,2}\right) s_{D}\left(h_{1,1,2}\right)+s_{D}\left(h_{2,1,1}\right) s_{D}\left(h_{1,1,2}\right)=0
$$

modulo 2.

A simple calculation reveals that there are 10 such stable orientations and 6 stable orientations that are irrelevant. Hence $\alpha$ equals average of 10 determinants of signings of $(3 \times 3)$ matrix $\mathcal{Z}$. We can check by hand that $\alpha=7 / 5$. Since $C_{r}=2$, we have

$$
-2^{C_{r}} x^{w(M)}+\alpha\left(2^{C_{r}}+1\right)=-4+5(7 / 5)=3 .
$$

If we want to use Theorem 1.1 to calculate $\mathcal{P}\left(Q_{3,3,2}, x\right)$ we would have $C_{r}=12$ and $\alpha$ equal the average of $2^{23}+2^{11}$ determinants of signings of a $(9 \times 9)$ matrix.

These huge numbers which appear even for very small lattices demonstrate the character of Theorem 1.1: it certainly does not aim to a computational efficiency.

Having the expression for the partition function of the dimer problem given by Theorem 1.1, let me briefly indicate how to transform the 3-dimensional Ising problem to the dimer problem of locally modified cubic lattice. This transformation goes back to Kasteleyn [13] and Fisher [2] and it is well described e.g. in [6]. An eulerian subgraph of a graph $G=(V, E)$ is a set of edges $U \subset E$ such that each vertex of $V$ is incident with an even number of edges from $U$. The generating function of eulerian subgraphs $\mathcal{E}(G, x)$ equals the sum of $x^{w(U)}$ over all eulerian subgraphs $U$ of $G$. The partition function of the Ising problem of a graph (with zero magnetic field) can be expressed as the generating function of eulerian subgraphs of the same graph, with modified edge weights. This classic relation between the Ising partition function and the generating function of eulerian subgraphs was discovered by van der Waerden:

$$
Z(\beta)=2^{n} \prod_{\{i, j\} \in E} \cosh \left(\beta J_{i j}\right) \quad \mathcal{E}\left(G, \tanh \left(\beta J_{i j}\right)\right)
$$

see [20]. Hence it remains to transform the generating function of eulerian subgraphs of the cubic lattice $Q_{m m k}$ into the generating function of perfect matchings of a locally modified graph $Q_{m m k}^{*}$. We use Fisher's construction [2] since it is local in the sense that it only modifies each vertex in a way dependent on its degree and it may be performed so that the embedding of $Q_{m m k}$ is preserved. Fisher's construction may be described as follows:

Let $G=(V, E)$ be a graph embedded in an orientable surface of genus $g$, and $v \in V$ a vertex. Let $e_{1}, e_{2}, \ldots, e_{d} \in E$ denote the edges incident with $v$, ordered clockwise as they spread out from $v$ in the embedding. Then the even splitting of $v$ is a graph $G^{\prime}=\left(V^{\prime}, E^{\prime}\right)$ where

$$
\begin{aligned}
& \text { - } V^{\prime}=V \backslash\{v\} \cup\left\{v_{1}, \ldots, v_{d}, v_{1}^{\prime}, \ldots, v_{d}^{\prime}\right\} \\
& \text { - } E^{\prime}=E \backslash\left\{e_{1}, e_{2}, \ldots, e_{d}\right\} \cup\left\{e_{1}^{\prime}, e_{2}^{\prime}, \ldots, e_{d}^{\prime}\right\} \cup E^{A}
\end{aligned}
$$




$$
\text { - } E^{A}=\left\{\left\{v_{i}, v_{i}^{\prime}\right\} ; i=1, \ldots, d\right\} \cup\left\{\left\{v_{i}, v_{i-1}^{\prime}\right\} ; i=2, \ldots, d\right\} \cup\left\{\left\{v_{i}^{\prime}, v_{i+1}^{\prime}\right\} ; i=1, \ldots, d-1\right\}
$$

The edges $e_{i}^{\prime} \in E^{\prime}$ (image edges) are obtained from $e_{i} \in E$ by replacing the vertex $v$ by $v_{i}$. The edges $E^{A}$ will be called auxiliary.

Note that the graph obtained by even splitting can be again embedded in the same surface since the transformation replaces a vertex $v \in V$ by a cluster of $2 d$ vertices and $3 d-2$ edges. The cluster itself is a planar graph which can be embedded in a small neighborhood of the original location of the vertex $v$. The images of the edges incident with $v$ can be embedded in the same way as they were in the original graph.

Let $G=(V, E)$ be a graph and $G^{*}=\left(V^{*}, E^{*}\right)$ the graph obtained by successive even splitting of all vertices in $V$. If there are weights $w(e)$ assigned to edges $e \in E$, we assign the same weights to their images in $E^{*}: w\left(e^{\prime}\right)=w(e)$. The auxiliary edges $f \in E^{*}$ get assigned $w(f)=0$. With this assigment of weights, the generating function of perfect matchings of $G^{*}$ is equal to the generating function of eulerian subgraphs of $G$,

$$
\mathcal{P}\left(G^{*}, x\right)=\mathcal{E}(G, x) .
$$

This may be observed as follows: if $M$ is a perfect matching in $G^{*}$, it must cover each of its vertices exactly once. Because the cluster replacing every vertex has an even number of vertices, and any of the auxiliary edges which is in $M$ covers a pair of vertices of the cluster, there remain an even number of vertices to be covered by the image edges incident with the cluster. Therefore, every cluster coincides with an even number of image edges which are in $M$; in other words, these edges form the image of an eulerian subgraph of $G$.

Vice versa, the image of any eulerian subgraph of $G$ can be extended (uniquely) by adding some of the auxiliary edges in $G^{*}$ to make a perfect matching in $G^{*}$. Thus, there is a one-to-one correspondence between the perfect matchings of $G^{*}$ and the eulerian subgraphs of $G$. As all the auxiliary edges have weights equal to 0 , the corresponding terms contributing to either of the generating functions are equal. Consequently, the two generating functions are equal.

Further in sections 2,3 we show how to calculate $\mathcal{P}\left(Q_{m m k}, x\right)$ by embedding $Q_{m m k}$ into a generalised surface $S_{g}$ so that $Q_{m m k}$ becomes a generalised g-graph. This embedding of $Q_{m m k}$ has a 'planar part' consisting of all the vertical edges, and this part doesnot play a role in the derivation of the formula, where the 'non-planar' edges are vital. The advantage of the Fisher's construction is that the even splitting of the vertices may be performed in the planar part of $Q_{m m k}$, hence the paths of vertical edges are replaced by the 'paths of triangles', and the non-planar part of $Q_{m m k}$ remains untouched. Hence $Q_{m m k}$ is turned into $Q_{m m k}^{*}$ without changing the embedding and an expression analogous to the one described in Corollory 3.9 for the dimer problem holds for the Ising problem as well.

In fact, one should find an analogous expression for the 3-dimensional variants of the problems which may be treated by the Pfaffian method in 2 dimensions, like a variant of the ice problem.

The proof of our result is involved: this paper may be viewed as a continuation of the papers [4], [5], [6], [7]. A theorem of Galluccio and Loebl [4] expresses $\mathcal{P}(G, x)$, where 
$G$ is an arbitrary graph, as a linear combination of Pfaffians of matrices associated with relevant orientations of $G$. When $G$ is a bipartite graph like the cubic lattice, the Pfaffians may be turned into determinants. The relevant orientations may be naturally described when the graph is embedded in a certain way on an orientable surface.

This 'Pfaffian approach' to the dimer problem has been started by Kasteleyn [13]. Kasteleyn [13] and Fisher [2] also described methods how to find the Ising partition function for a graph $\mathrm{G}$ as the dimer partition function of a locally modified $\mathrm{G}$. In [5] and [6], the Pfaffian method leads to an efficient algorithmic treatment of the Ising problem for finite lattices which may be embedded on a fixed surface, e.g. on a torus. This approach has been recently extended in [19] to non-orientable surfaces.

We use the Pfaffian method to prove Theorem 1.1 as follows: we embed the threedimensional cubic lattice to a 2-dimensional orientable surface, use the theory developed in [4] and finally characterize the coefficients of the resulting linear combination and turn it into a probabilistic expression.

Applying elementary probabilistic analysis to the statement of Theorem $1.1 \mathrm{I}$ have obtained a curious corollary which may be of independent interest. Once discovered, the corollary may be proved directly without using Theorem 1.1.

Let $Q^{\prime}$ be a cubic lattice with added boundary edges, i.e. the degree of each vertex of $Q^{\prime}$ is six. A subset $C$ of vertices of $Q^{\prime}$ is called a cover if each edge of $Q^{\prime}$ is incident with exactly one vertex of $C$. Note that $Q^{\prime}$ has exactly 2 covers. We fix one of them and denote it by $C$. A subgraph of $Q^{\prime}$ is called a plane if it is obtained from $Q^{\prime}$ by deleting both horizontal and/or vertical edges incident with each vertex of the cover $C$. Hence each vertex of $C$ has degree 2 or 4 in any plane. A plane $P$ is called even if the number of vertices of $C$ of degree 2 in $P$ is even, and $P$ is called odd otherwise.

\section{Theorem 1.2}

$$
\mathcal{P}\left(Q^{\prime}, x\right)=\sum_{W \in A} \mathcal{P}(W, x)-\sum_{W \in B} \mathcal{P}(W, x)
$$

where $A$ consists of the even planes and $B$ consists of the odd planes.

Proof. Let $M$ be a perfect matching of $Q^{\prime}$. We will compute how $M$ contributes to the RHS. Let $Z$ be the subset of vertices of $C$ incident to the width edges of $M$, and let $z=|Z|$. $M$ contributes to a term of the RHS corresponding to a plane $P$ if and only if $M$ is a perfect matching of $P$. Which planes contain $M$ ? Assume $M$ is a perfect matching of a plane $P$ and let $x \in C$. First let $x$ be incident with a horisontal or a vertical edge $e$ of $M$. Then the degree of $x$ in $P$ is 4 and $e$ determines which edges of $P$ are incident with $x$. Secondly let $x$ be incident with a width edge $e$ of $M$. Then all three possibilities may occur in $P: x$ may be incident with the width edges only, or with the width and the horisontal edges, or with the width and the vertical edges. Let $P$ have $i \leq z$ vertices of $Z$ incident with the width edges only. Then $M$ contributes $(-1)^{i}$ to the term of the RHS corresponding to $P$. Hence, the total contribution of $M$ equals $\sum_{i=0}^{z}(-1)^{i} 2^{z-i}\left(\begin{array}{c}z \\ i\end{array}\right)$, which equals $(2-1)^{z}=1$ by binomial theorem. 
The next section will describe a theorem of Galluccio and Loebl ([4]) which forms a basis of the proof of Theorem 1.1. The basic notation, definitions and some relevant simple facts may be found in the appendix.

\section{Generalized g-graphs.}

It is recommended to read the appendix first before starting with this section.

Definition 2.1 $A$ surface $S_{g}$ of genus $g$ consists of $a$ base $B_{0}$ and $2 g$ bridges $B_{j}^{i}, i=$ $1, \ldots, g$ and $j=1,2$, where

i) $B_{0}$ is a convex $4 g$-gon with vertices $a_{1}, \ldots, a_{4 g}$ numbered clockwise;

ii) $B_{1}^{i}, i=1, \ldots, g$, is a 4-gon with vertices $x_{1}^{i}, x_{2}^{i}, x_{3}^{i}, x_{4}^{i}$ numbered clockwise. It is glued with $B_{0}$ so that the edge $\left[x_{1}^{i}, x_{2}^{i}\right]$ of $B_{1}^{i}$ is identified with the edge $\left[a_{4(i-1)+1}, a_{4(i-1)+2}\right]$ of $B_{0}$ and the edge $\left[x_{3}^{i}, x_{4}^{i}\right]$ of $B_{1}^{i}$ is identified with the edge $\left[a_{4(i-1)+3}, a_{4(i-1)+4}\right]$ of $B_{0}$;

iii) $B_{2}^{i}, i=1, \ldots, g$, is a 4-gon with vertices $y_{1}^{i}, y_{2}^{i}, y_{3}^{i}, y_{4}^{i}$ numbered clockwise. It is glued with $B_{0}$ so that the edge $\left[y_{1}^{i}, y_{2}^{i}\right]$ of $B_{2}^{i}$ is identified with the edge $\left[a_{4(i-1)+2}, a_{4(i-1)+3}\right]$ of $B_{0}$ and the edge $\left[y_{3}^{i}, y_{4}^{i}\right]$ of $B_{2}^{i}$ is identified with the edge $\left[a_{4(i-1)+4}, a_{4(i-1)+5(\bmod 4 g)}\right]$ of $B_{0}$.

Observe that in Definition 2.1 we denote by $[a, b]$ edges of polygons and not edges of graphs. The usual representation in the space of an orientable surface $\mathcal{S}$ of genus $g$ may be then obtained from its polygonal representation $S_{g}$ by the following operation: for each bridge $B$, glue together the two segments which $B$ shares with the boundary of $B_{0}$, and delete $B$.

Definition 2.2 A graph $G$ is called a g-graph if it may be embedded on $S_{g}$ so that all the vertices belong to the base $B_{0}$, and the embedding of each edge uses at most one bridge. The set of the edges embedded entirely on the base will be denoted by $E_{0}$ and the set of the edges embedded on the bridge $B_{j}^{i}$ will be denoted by $E_{j}^{i}, i=1, \ldots, g, j=1,2$. We also let $G_{0}=\left(V, E_{0}\right)$ and $G_{j}^{i}=\left(V, E_{0} \cup E_{j}^{i}\right)$. Moreover the following conditions need to be satisfied too.

1. the outer face of $G_{0}=\left(V, E_{0}\right)$ is a cycle, and it is embedded on the boundary of $B_{0}$,

2. if $e \in E_{1}^{i}$ then $e$ is embedded entirely on $B_{1}^{i}$ and one end vertex of e belongs to $\left[x_{1}^{i}, x_{2}^{i}\right]$ and the other one belongs to $\left[x_{3}^{i}, x_{4}^{i}\right]$. Similarly, if $e \in E_{2}^{i}$ then $e$ is embedded entirely on $B_{2}^{i}$ and one end vertex of e belongs to $\left[y_{1}^{i}, y_{2}^{i}\right]$ and the other one belongs to $\left[y_{3}^{i}, y_{4}^{i}\right]$.

From now on, we shall consider $g$-graphs together with a fixed embedding on $S_{g}$. Given a $g$-graph $G$, we denote by $C_{0}$ the cycle which forms the outer face of $G_{0}$. 
Definition 2.3 Let $G$ be a g-graph and let $G_{j}^{i}=\left(V, E_{0} \cup E_{j}^{i}\right)$. If we draw $B_{0} \cup B_{j}^{i}$ on the plane as follows: $B_{0}$ along with the edges of the polygons belonging to its boundary is unchanged, and the edge $\left[x_{1}^{i}, x_{4}^{i}\right]$ ([ $\left[y_{1}^{i}, y_{4}^{i}\right]$ respectively) of $B_{j}^{i}$ is drawn so that it belongs to the external boundary of $B_{0} \cup B_{j}^{i}$, we obtain a planar embedding of $G_{j}^{i}$. This embedding will be called planar projection of $E_{j}^{i}$ outside $B_{0}$.

Definition 2.4 Let $G=(V, E)$ be a g-graph. An orientation $D_{0}$ of $G_{0}$ such that each inner face of each 2-connected component of $G_{0}$ is clockwise odd in $D_{0}$ is called a basic orientation of $G_{0}$.

Note that a basic orientation always exists for a planar graph. Kasteleyn [13] proved that if $D$ is a basic orientation of a planar graph $G$ then the contributions of all perfect matchings of $G$ have the same sign in $\operatorname{Pf}(A(D))$.

From now on we shall fix a basic orientation $D_{0}$ for each $g$-graph.

Definition 2.5 Let $G=(V, E)$ be a g-graph and $D_{0}$ a basic orientation of $G_{0}$. We define the orientation $D_{j}^{i}$ of each $G_{j}^{i}$ as follows: We consider $G_{j}^{i}$ embedded on the plane by the planar projection of $E_{j}^{i}$ outside $B_{0}$ (see Definition 2.3), and complete the basic orientation $D_{0}$ of $G_{0}$ to an orientation of $G_{j}^{i}$ so that each inner face of each 2-connected component of $G_{j}^{i}$ is clockwise odd.

The orientation $-D_{j}^{i}$ is defined by reversing the orientation $D_{j}^{i}$ of $G_{j}^{i}$.

Observe that after fixing a basic orientation $D_{0}$, the orientation $D_{j}^{i}$ is uniquely determined for each $i, j$.

Definition 2.6 Let $G$ be a g-graph, $g \geq 1$. An orientation $D$ of $G$ which equals the basic orientation $D_{0}$ on $G_{0}$ and which equals $D_{j}^{i}$ or $-D_{j}^{i}$ on $E_{j}^{i}$ is called relevant. We define its type $r(D) \in\{+1,-1\}^{2 g}$ as follows: For $i=0, \ldots, g-1$ and $j=1,2, r(D)_{2 i+j}$ equals +1 or -1 according to the sign of $D_{j}^{i+1}$ in $D$.

Definition 2.7 Let $G$ be a g-graph and $D$ a relevant orientation of $G$. Let $r(D)=$ $\left(r_{1}, \ldots, r_{2 g}\right)$. We let $c(r(D))$ equal the product of $c_{i}, i=0, \ldots, g-1$, where $c_{i}=c\left(r_{2 i+1}, r_{2 i+2}\right)$ and $c(1,1)=c(1,-1)=c(-1,1)=1 / 2$ and $c(-1,-1)=-1 / 2$.

Observe that $c(r(D))=(-1)^{n} 2^{-g}$, where $n=\left|\left\{i ; r_{2 i+1}=r_{2 i+2}=-1\right\}\right|$.

The following theorem is proved in Galluccio, Loebl [4]. See appendix for the definition of $s(D, M)$.

Theorem 2.8 Let $G$ be a g-graph with a perfect matching $M_{0} \subset E_{0}$. If we order the vertices of $G$ so that $s\left(D_{0}, M_{0}\right)$ is positive then

$$
\mathcal{P}(G, x)=\sum_{i=1}^{4^{g}} c\left(r\left(D_{i}\right)\right) \operatorname{Pf}\left(A\left(D_{i}\right)\right)
$$

where $D_{i}, i=1, \ldots, 4^{g}$, are the relevant orientations of $G$. 
We need a generalization of the notion of a $g$-graph.

Definition 2.9 Any graph $G$ obtained by the following construction will be called generalized g-graph.

1. Let $g=g_{1}+\ldots+g_{n}$ be a partition of $g$ into positive integers.

2. Let $S_{g_{i}}$ be a surface of genus $g_{i}, i=1, \ldots, n$. Let us denote the basis and the bridges of $S_{g_{i}}$ by $B_{0}^{i}$ and $B_{j, k}^{i}, i=1, \ldots, n, j=1, \ldots, g_{i}$ and $k=1,2$.

3. For $i=1, \ldots$, n let $H_{i}$ be a $g_{i}$-graph with the property that the subgraph of $H_{i}$ embedded on $B_{0}^{i}$ is a cycle, embedded on the boundary of $B_{0}^{i}$. Let us denote it by $C^{i}$.

4. Let $G_{0}$ be a 2-connected plane graph and let $F_{1}, \ldots, F_{n}$ be a subset of faces of $G_{0}$. Let $K^{i}$ be the cycle bounding $F_{i}, i=1, \ldots, n$. Let each $K^{i}$ be isomorphic to $C^{i}$.

5. Then $G$ is obtained by glueing the $H_{i}$ 's into $G_{0}$ so that each $K^{i}$ is identified with $C_{i}$.

For each generalized g-graph $G$ we can define $4^{g}$ relevant orientations $D_{1}, \ldots, D_{4^{g}}$ with respect to a fixed basic orientation of $G_{0}$, and coefficients $c\left(r\left(D_{i}\right)\right), i=1, \ldots, n$ in the same way as for a g-graph. The following theorem can be proved in the same way as Theorem 2.8 since each $H_{i}$ may be treated independently. In fact, G. Tessler chose this more general setting in his paper [19].

Theorem 2.10 Let $G$ be a generalized g-graph with a perfect matching $M_{0}$ of $G_{0}$. Let $D_{0}$ be a basic orientation of $G_{0}$. If we order the vertices of $G$ so that $s\left(D_{0}, M_{0}\right)$ is positive then

$$
\mathcal{P}(G, x)=\sum_{i=1}^{4^{g}} c\left(r\left(D_{i}\right)\right) \operatorname{Pf}\left(A\left(D_{i}\right)\right)
$$

where $D_{i}, i=1, \ldots, 4^{g}$, are the relevant orientations of $G$. 


\section{Cubic lattices as generalized g-graphs.}

In this section we will describe how to draw 3-dimensional cubic lattices as generalized g-graphs. Let $m, n$ be odd positive integers such that $k=(n-1) / 2$ is even. Let us use $Q$ to denote the cubic lattice $Q_{m, m, n}$. Let us denote vertical path $\left(V_{x y 1}, \ldots, V_{x y n}\right)$ of $Q$ by $V_{x y}(Q)=V_{x y}$ and let $\bar{V}_{x y}$ denote $V_{x y}$ traversed in the opposite direction.

Let $H_{x}(Q)=H_{x}=\left\{h_{x y z} ; z=1, \ldots, n, y=1, \ldots, m\right\}$ and $W_{x y}(Q)=W_{x y}=\left\{w_{x y z} ; z=\right.$ $1, \ldots n\}$.

\section{How to draw $Q$ on the plane.}

First draw the paths $V_{x y}$ along a cycle in the following natural way:

$$
V_{11}, \bar{V}_{12}, V_{13}, \ldots V_{1 m}, \bar{V}_{2 m}, V_{2(m-1)}, \ldots, \bar{V}_{21}, V_{31}, \ldots, V_{m m}
$$

Next, draw the horizontal edges inside this cycle, and the width edges outside of this cycle as depicted in Fig. 1 below where $Q=Q_{3,3,3}$ is properly drawn.

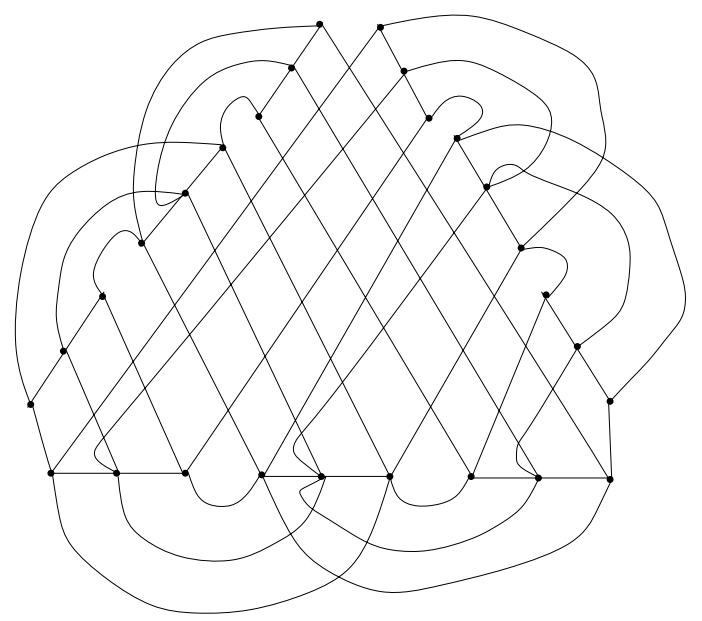

Figure 1

For each $x=1, \ldots, m-1$ the curves representing the edges of $H_{x}$ are pairwise disjoint and for $x=2, \ldots, m-2$ the curves representing the edges of $H_{x}$ intersect the curves representing the edges of $H_{x-1}$ and $H_{x+1}$. We keep the following rule: the interiors of the curves representing $h_{x y z}$ and $h_{(x+1) y z}$ intersect if and only if $z$ is even.

For each $x=1, \ldots, m$ and $y=1, \ldots,(m-1)$ the curves representing the edges of $W_{x y}$ are pairwise disjoint and for $y=2, \ldots, m-2$ the curves representing the edges of $W_{x y}$ intersect the curves representing the edges of $W_{x(y-1)}$ and $W_{x(y+1)}$. We again keep the rule that the interiors of the curves representing $w_{x y z}$ and $w_{x(y+1) z}$ intersect if and only if $z$ is even. The curve representing an edge $e$ will be denoted by $C(e)$.

Now we modify $Q$ into a generalized g-graph $Q^{\prime}$.

Width construction. First we describe the modification for $W_{x}, x=1, \ldots, m$. The modification is described by Fig. 2 where the construction is illustrated on edges among $V_{x(y-1)}, V_{x y}$ and $V_{x(y+1)}$ for $\mathrm{x}$ odd and $y<m-1$ even. 


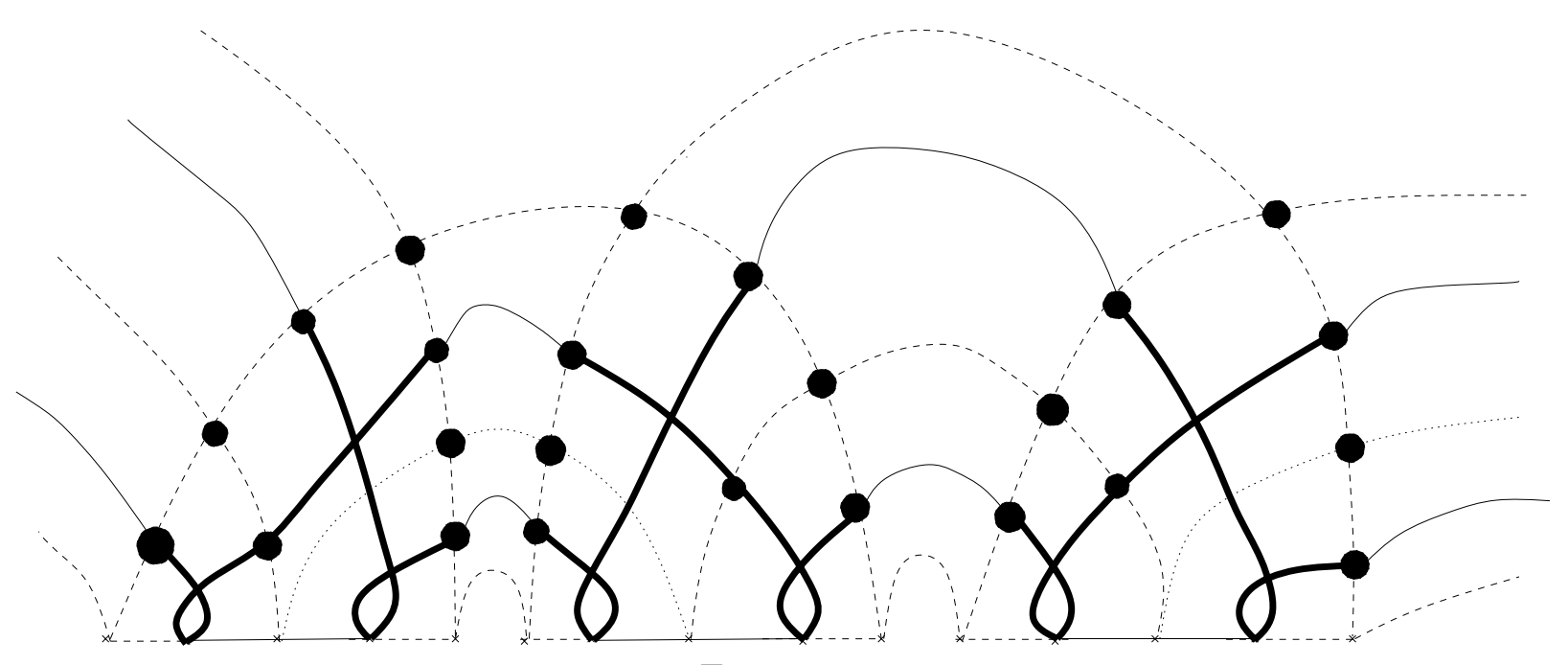

Figure 2

For each $x=1, \ldots, m$ perform the following construction:

1. For each $y$ even let $A u x_{1}=\left\{w_{x y z} ; z\right.$ odd $\}$. For each edge $e$ of $A u x_{1}$ introduce a new vertex to each intersection of $C(e)$ with the curves representing the edges of $W_{x(y-1)} \cup W$, where $W=W_{x(y+1)}$ in case $y<m-1$ and $W=\emptyset$ otherwise. By this operation, each $e \in A u x_{1}$ is replaced by a path. Call each edge of this path auxiliary.

2. For each $y$ even let $A u x_{2}=\left\{w_{x(y-1) 1}, w_{x(y-1) n}\right\} \cup A$, where $A=\left\{w_{x,(y+1) 1}, w_{x(y+1) n}\right\}$ in case $y<m-1$ and $A=\emptyset$ otherwise. For each edge $e$ of $A u x_{2}$ introduce a new vertex to each intersection of $C(e)$ with the curves representing the edges of $W_{x y}$. Hence each $e \in A u x_{2}$ is replaced by a path. Call each edge of this path auxiliary.

For each $y$ even the edges $v_{x y 1}, v_{x y(n-1)}$ and also $v_{x(y+1) 1}, v_{x(y+1)(n-1)}$ will also be called auxiliary.

In Figure 2, the auxiliary edges are represented by dashed lines.

3. We introduce a new variable $a$ which we associate with each auxiliary edge $e$ and we let $w(e)=1$. Hence the term associated with each auxiliary edge $e$ has form $a^{w(e)}=a$.

4. The edges $w_{x y z}, y$ even and $z$ even will be called relevant for $Q$. If $y<m-1$ then the relevant edges are subdivided by two vertices (added in 2.) into three edges of $Q^{\prime}$. The middle one will be called special and the other two long.

If $y=m-1$ then the relevant edge $w_{x y z}$ is subdivided by one vertex into two edges of $Q^{\prime}$. The one incident to $V_{x m}$ will be called special and the other one long.

If $e$ is a relevant edge of $Q$, then we choose a corresponding long edge $f$ and we let $w(e)=w(f)$. We let the weight of the special edge and of the remaining long edge be equal to 0 . 
5. The edges of $W_{x(y-1)} \cup W$ also got subdivided by new vertices introduced in step 2 and step 3 .

6. We delete all edges of the paths obtained from $w_{x(y-1) z}$ and $w_{x(y+1) z}, 1<z<n$ odd. In Figure 2, the deleted edges are represented by dotted lines.

7. Each edge $e \in\left\{w_{x(y-1) z}, w_{x(y+1) z} ; z\right.$ even $\}$, is subdivided by new vertices introduced in 2. into a path. We let the weights assigned to the edges of the path equal 0 except of one initial edge whose weight is let equal $w(e)$. The edge $e$ of this path such that the interior of $C(e)$ does not intersect interior of any curve representing a long edge will also be special.

8. All vertical edges which are not auxiliary (see 2.) will be called special.

In Figure 2, the special edges are represented by normal lines.

This finishes the construction for the width edges. In Figure 2, the edges which are neither auxiliary nor special nor deleted are represented by fat lines.

Horizontal construction. Now we perform an analogous construction with the horizontal edges of $Q$.

1. For each $x$ even let $A u x_{3}=\left\{h_{x y z} ; z\right.$ odd $\}$. For each edge $e$ of $A u x_{3}$ introduce a new vertex to each intersection of $C(e)$ with the curves representing the edges of $H_{x-1} \cup K$, where $K=H_{x+1}$ in case $x<m-1$ and $K=$ set otherwise. By this operation, each $e \in A u x_{3}$ is replaced by a path. Call each edge of this path auxiliary.

2. For each $x$ even let $A u x_{4}=\left\{h_{(x-1) 11}, h_{(x-1) n n}\right\} \cup B$, where $B=\left\{h_{(x+1) 11}, h_{(x+1) n n}\right\}$ in case $x<m-1$ and $B=\emptyset$ otherwise. For each edge $e$ of $A u x_{4}$ introduce a new vertex to each intersection of $C(e)$ with the curves representing the edges of $H_{x}$. Hence each $e \in A u x_{4}$ is replaced by a path. Call each edge of this path auxiliary.

3. We again associate variable a with each auxiliary edge $e$ and we let $w(e)=1$.

4. The edges $h_{x y z}, x$ even and $z$ even will be called relevant for $Q$. If $x<m-1$ then the relevant edges are subdivided by two vertices (added in 2.) into three edges of $Q^{\prime}$. The middle one will be called special and the other two long.

If $x=m-1$ then the relevant edge $h_{x y z}$ is subdivided by one vertex into two edges of $Q^{\prime}$. The one incident to $V_{m}$. will be called special and the other one long.

If $e$ is a relevant edge of $Q$, then we choose a corresponding long edge $f$ and we let $w(e)=w(f)$. We let the weight of the special edge and of the remaining long edge equal 0.

5. The edges of $H_{x-1} \cup K$ also got subdivided by new vertices introduced in step 2 and step 3 . 
6. We delete all edges of the paths obtained from $h_{(x-1) y z}$ and $h_{(x+1) y z}, 1<z<n$ odd.

7. Each edge $h \in\left\{h_{(x-1) y z}, h_{(x+1) y z} ; z\right.$ even $\}$, is subdivided by new vertices introduced in 2. into a path. We let the weights assigned to the edges of the path equal 0 except of one initial edge whose weight is let equal $w(h)$. Each edge $e$ of this path such that the interior of $C(e)$ does not intersect interior of any curve representing a long edge will also be special.

Final steps. Let $A u x$ denote the set of all auxiliary edges. Then $Q^{\prime}-A u x$ is a subdivision of $Q_{m m k}$. We subdivide some special edges so that the graph $\mathcal{Q}=Q^{\prime}-A u x$ is an even subdivision of $Q_{m m k}$. All these new edges will be special, and we set their weights equal 0.

The subgraph of $Q^{\prime}$ formed by the special edges consists of vertical paths (of odd length) and some other mutually disjoint paths which may have at most one vertex in common with the vertical paths. Hence there is matching $M_{0}^{\prime}$ of special edges covering all the vertices of the vertical paths, and all but possibly one vertex of each of the additional paths of special edges. We conclude the construction of $Q^{\prime}$ by subdividing some auxiliary edges in such a way that $Q^{\prime}$ has a perfect matching M' consisting of special and auxiliary edges only, which extends $M_{0}^{\prime}$ (i.e. $M_{0}^{\prime} \subset M^{\prime}$ ). All these new edges will be auxiliary; we again associate variable $a$ with them and we set their weights equal 1 .

This finishes the construction of $Q^{\prime}$.

\section{Some properties of $Q^{\prime}$.}

1. Each edge $e$ of $Q^{\prime}$ such that $C(e)$ does not intersect any curve representing other edge in its interior is auxiliary or special. Let us denote the plane subgraph of $Q^{\prime}$ formed by the auxiliary and special edges by $Q^{p}$.

2. Any other edge of $Q^{\prime}$ is drawn on a face of $Q^{p}$. Moreover, the edges drawn on a face of $Q^{p}$ may be drawn onto a pair of bridges above this face, where one of the bridges contains one long edge, and the other bridge contains the remaining edges. Hence, we may view $Q^{\prime}$ as a generalized g-graph with the planar part equaled to $Q^{p}$.

3 The special edges form an acyclic subgraph of $Q^{p}$ (see Fig. 2). Hence any orientation of the special edges may be extended into a basic orientation of $Q^{p}$. We will choose basic orientation $D^{p}$ of $Q^{p}$ with the following properties:

1. $D^{p}$ on special edges is in agreement with the natural ordering,

2. Perfect matching $M^{\prime}$ has positive sign in $\operatorname{Pf}\left(A\left(D^{p}\right)\right)$,

3. The orientation of edges on a bridge has positive sign if and only if it is in agreement with the natural ordering $\left(V_{11} \bar{V}_{12} \ldots . V_{1 m} \bar{V}_{21} \ldots . V_{m m}\right)$.

4. We constructed $Q^{\prime}$ so that $\mathcal{Q}=Q^{\prime}-A u x$ is an even subdivision of $Q_{m m k}$. If $w$ is a vector of weights associated with $Q_{m m k}$ then let $w^{\prime}$ be the vector of weights associated with $\mathcal{Q}$ and induced by $w$ and let $w^{\prime \prime}$ be the vector of weights associated with $Q^{\prime}$ which equals $w^{\prime}$ on the edges of $\mathcal{Q}$ and $w^{\prime \prime}(e)=1$ for each auxiliary edge $e$ of $Q^{\prime}$. If we let $a=0$ we have $\mathcal{P}\left(Q^{\prime}, x, a\right)=\mathcal{P}\left(Q_{m m k}, x\right)$. 
We have described how to view $Q_{m m k}, m$ odd and $k$ even, as a generalized g-graph $Q^{\prime}$. Now we can use Theorem 2.10 for $Q^{\prime}$ to compute $\mathcal{P}\left(Q_{m m k}, x\right)$.

\section{The relevant orientations of $Q^{\prime}$.}

Each relevant edge of $Q$ corresponds to unique edge of $Q_{m m k}$; this unique edge will also be called relevant in $Q_{m m k}$. Hence the relevant edges of $Q_{m m k}$ are: $w_{x y z}\left(Q_{m m k}\right)$, $x=1, \ldots, m, y$ even and and $h_{x y z}\left(Q_{m m k}\right), x$ even. Hence there are $1 / 2 k m(m-1)$ relevant width edges and 1/2(m-1)mk relevant horizontal edges in $Q_{m m k}$.

We let $\mathcal{R}$ be the set of relevant edges of $Q_{m m k}$ and $C_{r}=|\mathcal{R}|=k m(m-1)$ denote the number of relevant edges of $Q_{m m k}$.

The set $\mathcal{S}$ of the edges of $V_{i j}\left(Q_{m m k}\right), i, j=1, \ldots m$, corresponds to a subset of special edges of $Q^{\prime}$. The orientation $D^{p}$ induces orientation $\mathcal{S}^{d}$ of $\mathcal{S}$ which is in agreement with the natural ordering $\left(V_{11}\left(Q_{m m k}\right) \bar{V}_{12}\left(Q_{m m k}\right) \ldots V_{1 m}\left(Q_{m m k}\right) \bar{V}_{21}\left(Q_{m m k}\right) \ldots . . V_{m m}\left(Q_{m m k}\right)\right)$.

Each relevant orientation $D^{\prime}$ of $Q^{\prime}$ is determined by the fixed basic orientation $D^{p}$ of $Q^{p}$, and by a pair of signs for each pair of bridges. Each pair of bridges is associated with a long edge of $Q^{\prime}$. Hence these signs may be given by specifying $\left(d_{D^{\prime}}^{1}(e), d_{D^{\prime}}^{2}(e)\right) \in\{+-\}^{2}$, for each long edge $e$, where $d_{D^{\prime}}^{1}(e)$ denotes the sign of the bridge containing $e$, and $d_{D^{\prime}}^{2}(e)$ denotes the sign of the other bridge.

The long edges of $Q^{\prime}$ are associated with relevant edges of $Q$, and hence also with relevant edges of $Q_{m m k}$.

The relevant edges $w_{x(m-1) z}\left(Q_{m m k}\right)$ and $h_{(m-1) y z}\left(Q_{m m k}\right)$ are associated with only one long edge of $Q^{\prime}$. If $e$ is such relevant edge of $Q_{m m k}$, we will call $e$ border edge and we denote by $e_{1}$ the corresponding long edge. We let $d_{D^{\prime}}(e)=\left(d_{D^{\prime}}^{1}\left(e_{1}\right), d_{D^{\prime}}^{2}\left(e_{1}\right),+,+\right)$.

Let $C_{b}=2 m k$ denote the number of border edges.

Each relevant non-border edge $e$ of $Q_{m m k}$ has two long edges $e_{1}, e_{2}$ associated with it. We let $\left.d_{D^{\prime}}(e)=\left(d_{D^{\prime}}^{1}\left(e_{1}\right), d_{D^{\prime}}^{2}\left(e_{1}\right), d_{D^{\prime}}^{1}\left(e_{2}\right), d_{D^{\prime}}^{2}\left(e_{2}\right)\right)\right)$.

A relevant vector is any element $r$ of $\left[\{+,-\}^{4}\right]^{\mathcal{R}}$ such that $r(e)_{3}=r(e)_{4}=+$ for each relevant border edge $e$ of $Q_{m m k}$. Hence there are $4^{2 C_{r}-C_{b}}$ relevant vectors.

There is a natural bijection between relevant orientations of $Q^{\prime}$ and relevant vectors. If $s$ is a relevant vector, then let $D^{\prime}(s)$ denote the corresponding relevant orientation of $Q^{\prime}$ and let $\operatorname{sgn}(s)$ of a relevant vector $s$ be calculated according to Theorem 2.10 as follows: $\operatorname{sgn}(s)=(-1)^{\left|\left\{(e, i) ; i=0,1, s(e)_{2 i+1}=s(e)_{2 i+2}=-1\right\}\right|}$.

If $D^{\prime}(s)$ is a relevant orientation of $Q^{\prime}$ then let $D(s)$ denote the orientation of $Q_{m m k}$ induced by $D^{\prime}(s)$ (see 5.4). An orientation of $Q_{m m k}$ will be called relevant if it equals $D(s)$ for some relevant vector $s$.

Let $M$ be the (uniquely determined) perfect matching of $Q_{m m k}$ consisting of the edges of $\mathcal{S}$ only. Recall that perfect matching $M^{\prime}$ has positive sign in $\operatorname{Pf}\left(A\left(D^{p}\right)\right)$ and similarly, perfect matching $\mathrm{M}$ has positive sign in $\operatorname{Pf}\left(A\left(\mathcal{S}^{d}\right)\right)$. 
Using Theorem 2.10 and Theorem 5.5 we have the following.

\section{Theorem 3.1}

$$
\mathcal{P}\left(Q_{m m k}, x\right)=2^{-2 C_{r}+C_{b}} \sum \operatorname{sgn}(s) \operatorname{Pf}(A(D(s)))
$$

where the sum is over all relevant vectors.

Note that possibly $D(r)=D\left(r^{\prime}\right)$ for relevant vectors $r \neq r^{\prime}$. Next we clarify this.

Definition 3.2 We define an equivalence $*$ on the relevant vectors as follows. $r * s$ if the following holds: there is exactly one relevant non-border edge e such that $r(e) \neq s(e)$ and $r(f)=s(f)$ for each $f \neq e$. Moreover, $r(e)_{1} \neq s(e)_{1}, r(e)_{3} \neq s(e)_{3}, r(e)_{2}=s(e)_{2} \neq$ $r(e)_{4}=s(e)_{4}$.

Proposition 3.3 If $r * s$ then $D(r)=D(s)$ and $\operatorname{sgn}(r) \neq \operatorname{sgn}(s)$.

Proof. If $r * s$ then $D(r)=D(s)$ by the definition of ' ${ }^{*}$ '. Moreover $\operatorname{sgn}(r) \neq \operatorname{sgn}(s)$ since $r(e)_{2}=s(e)_{2} \neq r(e)_{4}=s(e)_{4}$ where $e$ is the only relevant edge for which $r(e) \neq s(e)$.

Definition 3.4 A relevant vector $r$ is called useful if it forms a one-element class w.r.t. equivalence $*$, i.e. if $r(e)_{2}=r(e)_{4}$ for each relevant non-border edge e.

\section{Corollary 3.5}

$$
\mathcal{P}\left(Q_{m m k}, x\right)=2^{-2 C_{r}+C_{b}} \sum \operatorname{sgn}(r) \operatorname{Pf}(A(D(r)))
$$

where the sum is over all useful vectors $r$.

Definition 3.6 If $r, s$ are useful vectors we write $r * * s$ if $D(r)=D(s)$.

Proposition 3.7 1. Each equivalence class of '**' has $2^{C_{r}-C_{b}}$ elements.

2. If $r * * s$ then $\operatorname{sgn}(r)=\operatorname{sgn}(s)$.

Proof. Let $r$ be a useful vector. Then $D(r)$ determines uniquely $r(e)_{2}$ and $r(e)_{4}$ for each relevant edge $e$ and also $r(f)_{1}$ for each relevant border edge $f$. Hence $D(r)$ determines uniquely $r(f)$ for each relevant border edge $f$. Moreover $D(r)$ determines uniquely the product $r(e)_{1} \times r(e)_{3}$ for each relevant non-border edge $e$. Since there are $C_{r}-C_{b}$ relevant non-border edges, each equivalence class of '**' has $2^{C_{r}-C_{b}}$ elements.

Let $r, s$ be useful and let $r * * s$. Then $r(e)_{2}=s(e)_{2}=s(e)_{4}=r(e)_{4}$ for each relevant non-border edge $e$ and $r(e)_{2}=s(e)_{2}$ and $s(e)_{1}=r(e)_{1}$ for each relevant border edge. This implies that $\operatorname{sgn}(r)=\operatorname{sgn}(s)$. 
Proposition 3.8 If $D$ is an orientation of $Q_{m m k}$ that extends $\mathcal{S}^{d}$ then there is uniquely determined class $C$ of equivalence $* *$ such that $D=D(r)$ for each $r \in C$.

Hence, given an orientation $D$ of $Q_{m m k}$ that extends $\mathcal{S}^{d}$, let us call it stable orientation and let us define its $\operatorname{sign} \operatorname{sgn}(D)$ to be equal to $\operatorname{sgn}(r)$ for any useful vector $r$ such that $D=D(r)$. This is well defined by Proposition 3.7.

\section{Corollary 3.9}

$$
\mathcal{P}\left(Q_{m m k}, x\right)=2^{-C_{r}} \sum \operatorname{sgn}(D) \operatorname{Pf}(A(D))
$$

over all stable orientations $D$.

We continue by characterizing $\operatorname{sgn}(D)$.

As we noticed before, $\operatorname{sgn}(r)=(-1)^{\left|\left\{(e, i) ; i=0,1, r(e)_{2 i+1}=r(e)_{2 i+2}=-1\right\}\right|}$. If $r$ is a stable vector then $r(e)_{2}=r(e)_{4}$ for each relevant non-border edge $e$ and we get the following observation.

Proposition 3.10 Let $r$ be a stable vector. Then $\operatorname{sgn}(r)=(-1)^{\left|\left\{e ; r(e)_{1} r(e)_{3}=-1, r(e)_{2}=-1\right\}\right|}$.

Definition 3.11 Let $D$ be a stable orientation. We define orientation $\bar{D}$ as follows:

1. For each $x, y, z$ such that $y<m$ odd do the following: let $n(x y z)$ be the number of arcs $w_{x a b}, a \leq y$ odd and $z \leq b \leq(z+1)$, oriented in $D$ against the natural ordering. If $n(x y z)$ odd then we orient $w_{x y z}$ in $\bar{D}$ against the natural ordering, else according to the natural ordering.

2. For each $x, y, z$ such that $x<m$ odd do the following: let $n(x y z)$ be the number of arcs $h_{a b c}$ oriented in $D$ against the natural ordering. Here $(a b c)$ are the triples of indices satisfying $a \leq x$ odd and $(y, z) \leq(b, c) \leq\left(y^{\prime}, z^{\prime}\right)$ where the order is lexicographic and $\left(y^{\prime}, z^{\prime}\right)$ is immediate successor of $(y, z)$.

If $n(x y z)$ odd then we orient $h_{x y z}$ in $\bar{D}$ against the natural order, else according to the natural order.

3. All the remaining arcs orient in $\bar{D}$ in the same way as in $D$.

Note that relevant edges are oriented in the same way in both $D$ and $\bar{D}$. 
Proposition 3.12 Let $D$ be a stable orientation and let $r$ be a stable vector such that $D=D(r)$. Let e be a relevant edge of $Q_{m m k}$. Then

1. $r(e)_{1} \times r(e)_{3}=-1$ if and only if $e$ is oriented in $D$ (and hence also in $\bar{D}$ ) against the natural ordering.

2. If $e=w_{x y z}, y$ even then $r(e)_{2}=-1$ if and only if $w_{x(y-1) z}$ is oriented in $\bar{D}$ against the natural ordering. If $e=h_{x y z}, x$ even then $r(e)_{2}=-1$ if and only if $h_{(x-1) y z}$ is oriented in $\bar{D}$ against the natural ordering.

Proof. $r(e)_{1} \times r(e)_{3}=-1$ if and only if exactly one long edge of $Q^{\prime}$ corresponding to $e$ is oriented in $D^{\prime}$ (we remind that $D$ is induced by orientation $D^{\prime}$ of $Q^{\prime}$ ) against the natural ordering. Since $D$ is induced by $D^{\prime}$, this happens if and only if $e$ is oriented in both $D$ and $\bar{D}$ against the natural ordering.

It remains to prove 2 . We will show the case $e=w_{x y z}$ since the other case is completely analogous. If $f$ is an edge of $Q_{m m k}$ then we let $f(D)=1$ if $f$ is oriented in $D$ according to the natural order, and we let $f(D)=-1$ otherwise. We proceed by induction on $(y, k-z)$.

Firstly assume $y=2$ and $z=k$. In this simplest case $r(e)_{2}=w_{x 1 k}(D)$ (see Fig. 2). Moreover the orientation of $w_{x 1 k}$ is the same in both $D$ and $\bar{D}$.

Secondly let $y=2$ and $z<k$. Then $w_{x 1 z}(D)=w_{x 1(z+1)}(D) \times r(e)_{2}$ (see Fig. 2). It follows from Definition 3.11 that $r(e)_{2}=-1$ if and only if $w_{x 1 z}$ is oriented in $\bar{D}$ against the natural order.

Thirdly, let $y=4$ and $z=k$. Then $w_{x 3 k}(D)=w_{x 1 k}(D) \times r(e)_{2}$ (see Fig. 2). It follows from Definition 3.11 that $r(e)_{2}=-1$ if and only if $w_{x 3 k}$ is oriented in $\bar{D}$ against the natural order.

Fourthly, let $y=4$ and $z<k$. Then $w_{x 3 z}(D)=w_{x 3(z+1)}(D) \times r(e)_{2} \times r\left(w_{x 2 z}\right)_{2}=$ $w_{x 3(z+1)}(D) \times r(e)_{2} \times w_{x 1 z}(D) \times w_{x 1(z+1)}(D)$ (see Fig. 2). It follows from Definition 3.11 that $r(e)_{2}=-1$ if and only if $w_{x 3 z}$ is oriented in $\bar{D}$ against the natural ordering.

In general if $e=w_{x y z}, y$ even and $z=k$ then $w_{x(y-1) k}(D)=r\left(w_{x(y-2) k}\right)_{2} \times r(e)_{2}=$ $r(e)_{2} \times \prod\left(w_{x y^{\prime} k}(D) ; y^{\prime}<y-1\right.$ odd $)$. It follows from Definition 3.11 that $r(e)_{2}=-1$ if and only if $w_{x(y-1) k}$ is oriented in $\bar{D}$ against the natural order.

Finally if $e=w_{x y z}, y$ even and $z<k$ then $w_{x(y-1) z}(D)=w_{x(y-1)(z+1)}(D) \times r(e)_{2} \times$ $r\left(w_{x(y-2) z}\right)_{2}=w_{x(y-1)(z+1)}(D) \times r(e)_{2} \times \prod\left(w_{x y^{\prime} z}(D) ; y^{\prime}<y-1\right.$ odd $) \times \prod\left(w_{x y^{\prime}(z+1)}(D) ; y^{\prime}<\right.$ $y-1$ odd $)$. It follows from Definition 3.11 that $r(e)_{2}=-1$ if and only if $w_{x 3 z}$ is oriented in $\bar{D}$ against the natural order.

Corollary 3.13 Let $D$ be a stable orientation. Then $\operatorname{sgn}(D)=(-1)^{h+\sum_{x=1}^{m} w(x)}$, where $w(x)=\mid\left\{(y z) ; y\right.$ even and both $w_{x y z}, w_{x,(y-1), z}$ are oriented against the natural ordering in $\bar{D}\}|; h=|\left\{(x y z) ; x\right.$ even and both $w_{x y z}, w_{(x-1), y, z}$ are oriented against the natural ordering in $\bar{D}\} \mid$. 
We can also write the sign in the following form: for edge $e$ we let $s_{D}(e)=0$ if the orientation of $e$ agrees with the natural order, and $s_{D}(e)=1$ otherwise.

Corollary 3.14 Let D be a stable orientation. Then

$$
\operatorname{sgn}(D)=(-1)^{\sum_{A} s_{D}\left(w_{x, 2 y, z}\right) s_{D}\left(w_{x, 2 y^{\prime}-1, z^{\prime}}\right)+\sum_{B} s_{D}\left(h_{2 x, y, z}\right) s_{D}\left(h_{2 x^{\prime}-1, y^{\prime}, z^{\prime}}\right)}
$$

where

$A=\left\{\left(x, y, z, y^{\prime}, z^{\prime}\right) ; 1 \leq x \leq m, 1 \leq y \leq(m-1) / 2,1 \leq z \leq k, 1 \leq y^{\prime} \leq y, z \leq z^{\prime} \leq z+1\right\}$

and

$$
\begin{gathered}
B=\left\{\left(x, y, z, x^{\prime}, y^{\prime}, z^{\prime}\right) ; 1 \leq x \leq(m-1) / 2,1 \leq z \leq k, 1 \leq y \leq m, 1 \leq x^{\prime} \leq x,\right. \\
\left.(y, z) \leq\left(y^{\prime}, z^{\prime}\right) \leq\left(y^{\prime \prime}, z^{\prime \prime}\right)\right\} .
\end{gathered}
$$

In the definition of $B$ the order on pairs of integers is lexicographic order and $\left(y^{\prime \prime}, z^{\prime \prime}\right)$ is the immediate successor of $(y, z)$.

Proposition 3.15 There are $2^{2 C_{r}}$ stable orientations. There are $2^{C_{r}-1}\left(2^{C_{r}}+1\right)$ stable orientations with positive sign.

Proof. The first statement follows directly from the definition of a stable orientation. For $Q_{132}$ there are $16=2^{4}=2^{2 C_{r}}$ stable orientations (see the remark after Theorem 1.1 for the definition of $C_{r}$ ), from which 10 have positive sign. Hence the difference between the number of stable orientations of positive sign and stable orientations of negative sign is $4=2^{C_{r}}$. For $Q_{152}$ there are $4^{4}=2^{2 C_{r}}$ stable orientations from which $10 \times 10+6 \times 6$ have positive sign and $2(6 \times 10)=120$ have negative sign. Hence the difference between the number of stable orientations of positive sign and stable orientations of negative sign is $10 \times 10+6 \times 6-2(6 \times 10)=(10-6)(10-6)=2^{C_{r}}$. Similarly by induction on $a$ we get that for $Q_{1(2 a+1) 2}$ there are $4^{2 a}=2^{2 C_{r}}$ stable orientations, and the difference between the number of stable orientations of positive sign and stable orientations of negative sign is $2^{2 a}$. Similarly we calculate the difference for $Q_{13 k}, k$ even, and by induction also for $Q_{1 m k}$. That takes care of one layer of width edges, and the layers are independent so the differences multiply as indicated above. In the same way we calculate the contribution of the horisontal edges. Summarising for $Q_{m m k}$ the difference between the number of stable orientations of positive sign and those of negative sign equals $2^{C_{r}}=2^{(m-1) k m}$. From this Proposition follows.

\section{From Pfaffians to determinants.}

In the introduction we let $\mathcal{Z}$ be square $\left(Z_{1} \times Z_{2}\right)$ matrix defined by $\mathcal{Z}_{i j}=x_{i j}$ if $i j$ is an edge of $Q_{m m k}$ and $\mathcal{Z}_{i j}=0$ otherwise. 
Let $D$ be an orientation of $Q_{m m k}$. In the introduction we associate a signing $Z(D)$ of $\mathcal{Z}$ with it such that $Z(D)_{i j}=x_{i j}$ if $(i j) \in E(D), Z(D)_{i j}=-x_{i j}$ if $(j i) \in E(D)$, and $Z(D)_{i j}=0$ otherwise.

Note that $P f(A(D))=\operatorname{det}(Z(D))$. Hence we can reformulate Corollary 3.9:

Corollary 4.1 $\mathcal{P}\left(Q_{m m k}, x\right)=2^{-C_{r}} \sum \operatorname{sgn}(D) \operatorname{det}(Z(D))$ where the sum is over all stable orientations $D$ of $Q_{m m k}$.

Recall that $M$ is unique perfect matching consisting only of vertical edges.

Proposition 4.2 The average of $\operatorname{det}(Z(D))$, D stable, equals $x^{w(M)}$.

Proof. By the linearity of expectation and the definition of stable orientations, the contribution of other than vertical edges cancel out when we calculate the average of $\operatorname{det}(Z(D)), D$ stable. Since $Q_{m m k}$ has exactly one perfect matching consisting of vertical edges only, Proposition follows.

Proof of Theorem 1.1. By Corollary 4.1 and Proposition 3.15 we have that $\mathcal{P}\left(Q_{m m k}, x\right)=2^{-C_{r}}\left[-2^{2 C_{r}} x^{w(M)}+2 \alpha\left(2^{C_{r}-1}\left(2^{C_{r}}+1\right)\right]=-2^{-C_{r}+2 C_{r}} x^{w(M)}+\alpha\left(2^{C_{r}}+1\right)\right.$.

Conclusion. We have expressed the partition functions of the dimer problem and the Ising problem in 3-dimensional finite cubic lattices by means of expectations of the determinants of matrices associated with the cubic lattices. This may open a possibility to apply fundamentally different statistical methods and Monte Carlo simulations to study these problems.

\section{References}

[1] A. Cayley. Sur les determinants gauches. Crelle's J., 38:93-96, 1847.

[2] M.E. Fisher. On the dimer solution of planar Ising models. Journal of Mathematical Physics, 7,10:1776, 1966.

[3] R.H. Fowler and G.S. Rushbrooke. Trans. Faraday Soc., 33:1272, 1937.

[4] A. Galluccio and M. Loebl. A theory of pfaffian orientations I: Perfect matchings and permanents. Electronic Journal of Combinatorics, 6,1, 1999.

[5] A. Galluccio and M. Loebl. A theory of pfaffian orientations II: T-joins, k-cuts and duality of enumeration. Electronic Journal of Combinatorics, 6,1, 1999.

[6] A. Galluccio and M. Loebl J. Vondrak. A new algorithm for the ising problem. Physical Review Letters, 84,26:5924-5927, 2000. 
[7] A. Galluccio and M. Loebl J. Vondrak. Optimization via enumeration: a new algorithm for the max cut problem. Mathematical Programming, 90:273-290, 2001.

[8] V.L. Girko. Random Matrices. Handbook of Algebra 1, North Holland, Amsterdam, 1996.

[9] O.J. Heilmann and E.H. Lieb. Monomers and dimers. Phys. Rev. Letters, 24:1412$1414,1970$.

[10] O.J. Heilmann and E.H. Lieb. Theory of monomer dimer systems. Comm. Math. Phys., 25:190-232, 1972.

[11] M.E. Fisher H.N.V. Temperley. Phil. Mag. Serie 8, 6, 1961.

[12] S. Istrail. Statistical mechanics, three-dimensionality and np-completeness. In Proceedings of the annual ACM symposium on the theory of computing (STOC), pages $87-96,2000$.

[13] P. W. Kasteleyn. The statistics of dimers on a lattice. Physica, 27:1209-1225, 1961.

[14] P.W. Kasteleyn. Graph theory and crystal physics. In Graph theory and theoretical physics, New York, 1967. Academic Press.

[15] L. Lovasz and M.D. Plummer. Matching Theory. Annals of Discrete Mathematics, 1986.

[16] T. Regge and R. Zecchina. Exact solution of the ising model on group lattices of genus $g>1$. J. Math. Phys., 37:2796, 1996.

[17] T. Regge and R. Zecchina. Combinatorial and topological approach to the 3d ising model. J. Phys. A, 33:741-761, 2000.

[18] J.K. Roberts. Proc. Roy. Soc. (London) A, 161:141, 1935.

[19] G. Tesler. Matchings in graphs on non-orientable surfaces. Journal of Comb. Theory $B, 78: 198-231,2000$.

[20] B.L. van der Waerden. Die lange reichweite der regelmassigen atomanordnung in mischkristallen. Z.Physik, 118:473, 1941.

\section{Appendix: Basic notation, definitions and facts.}

Let $G=(V, E)$ be a graph. We will assume that a weight $w(e)$ is associated with each edge e of G. If $A \subset E$ then we let $w(A)=\sum_{e \in A} w(e)$. A graph $G^{\prime}=\left(V^{\prime}, E^{\prime}\right)$ is called a subgraph of $G$ if $V^{\prime} \subset V$ and $E^{\prime} \subset E$. Let $\left\{v_{1}, e_{1}, v_{2}, e_{2}, \ldots, v_{i}, e_{i}, v_{i+1}, \ldots, e_{n}, v_{n+1}\right\}$ be a sequence such that each $v_{j}$ is a vertex of a graph $G$, each $e_{j}$ is an edge of $G$ and $e_{j}=v_{j} v_{j+1}$, and $v_{i} \neq v_{j}$ for $i<j$ except if $i=1$ and $j=n+1$. If also $v_{1} \neq v_{n+1}$ then $P$ is called $a$ 
path of $G$. If $v_{1}=v_{n+1}$ then $P$ is called a cycle of $G$. In both cases the length of $P$ equals $n$. When no confusion arises we shall also denote paths by simply listing their vertices or edges, namely $P=\left(v_{1}, v_{2}, \ldots, v_{n+1}\right)$ or $P=\left(e_{1}, e_{2}, \ldots, e_{n}\right)$.

A graph $G=(V, E)$ is connected if it has a path between any pair of vertices, and it is 2-connected if the graph $G_{v}=(V-\{v\},\{e \in E ; v \notin e\})$ is connected for each vertex $v$ of $G$. Each maximal 2-connected subgraph of $G$ is called a 2-connected component of $G$.

A graph $G^{\prime}$ is a subdivision of a graph $G$ if some edges of $G$ are replaced in $G^{\prime}$ by paths so that the inner vertices of each such new path have all degree 2 in $G^{\prime}$, and both terminal vertices coincide with the vertices of the corresponding deleted edge of $G$. $G^{\prime}$ is an even subdivision of $G$ if the new paths all have odd lengths. Let $w$ be the vector of the weights associated with the edges of $G$. We define induced weights $w^{\prime}$ for $G^{\prime}$ as follows: if $e$ is an edge of $G$ which is replaced by path $\left(e_{1}, \ldots, e_{n}\right)$ in $G^{\prime}$ consisting of $n$ edges then $w^{\prime}\left(e_{1}\right)=w(e), w^{\prime}\left(e_{j}\right)=0$ for each $j>1$ and $w^{\prime}(f)=w(f)$ for the remaining edges $f$ of $G$.

Let $G=(V, E)$ be a graph with $2 n$ vertices and $D$ an orientation of $G$. Denote by $A(D)$ the skew-symmetric matrix with the rows and the columns indexed by $V$, where $a_{u v}=x^{w(u, v)}$ in case $(u, v)$ is an $\operatorname{arc}$ of $D, a_{u, v}=-x^{w(u, v)}$ in case $(v, u)$ is an $\operatorname{arc}$ of $D$, and $a_{u, v}=0$ otherwise.

The Pfaffian of $A(D)$ is defined as

$$
P f(A(D))=\sum_{P} s^{*}(P) a_{i_{1} j_{1}} \cdots a_{i_{n} j_{n}}
$$

where $P=\left\{\left\{i_{1} j_{1}\right\}, \cdots,\left\{i_{n} j_{n}\right\}\right\}$ is a partition of the set $\{1, \ldots, 2 n\}$ into pairs, $i_{k}<j_{k}$ for $k=1, \ldots, n$, and $s^{*}(P)$ equals the sign of the permutation $i_{1} j_{1} \ldots i_{n} j_{n}$ of $12 \ldots(2 n)$.

Each nonzero term of the expansion of the Pfaffian of $A(D)$ equals $x^{w(P)}$ or $-x^{w(P)}$ where $P$ is a perfect matching of $G$. If $s(D, P)$ denote the sign of the term $x^{w(P)}$ in the expansion, we may write

$$
P f(A(D))=\sum_{P} s(D, P) x^{w(P)} .
$$

The Pfaffian is a determinant-type expression. Note the following classic result of Cayley (see [1]).

Theorem 5.1 Let $G$ be a graph and let $D$ be an orientation of $G$. Then

$$
P f^{2}(A(D))=\operatorname{det}(A(D)) .
$$

Let $A \Delta B$ denote the symmetric difference of the sets $A$ and $B$ and let $a \stackrel{2}{=} b$ denote $a=b$ modulo 2 .

Let $M, N$ be two perfect matchings of a graph $G$. Then $M \Delta N$ consists of vertex disjoint cycles of even length. These cycles are called alternating cycles of $M$ and $N$.

Let $C$ be a cycle of $G$ of an even length and let $D$ be an orientation of $G$. $C$ is said to be clockwise even in $D$ if it has an even number of edges directed in $D$ in agreement with the clockwise traversal. Otherwise $C$ is called clockwise odd. 
Definition 5.2 Let $G$ be a graph and let $D$ be an orientation of $G$. Let $M$ be a perfect matching of $G$. For each perfect matching $P$ of $G$ let $\operatorname{sgn}(D, M \Delta P)=(-1)^{n}$ where $n$ is the number of clockwise even alternating cycles of $M$ and $P$, and let $\mathcal{P}(D, M)$ be the sum of $\operatorname{sgn}(D, M \Delta P) x^{w(P)}$ over all perfect matchings $P$ of $G$.

The following theorem was proved by Kasteleyn [13].

Theorem 5.3 Let $G$ be a graph and $D$ an orientation of $G$. Let $P, M$ be two perfect matchings of $G$. Then

$$
s(D, P)=s(D, M) \operatorname{sgn}(D, M \Delta P) .
$$

Hence,

$$
P f(A(D))=\sum_{P} s(D, P) x^{w(P)}=s(D, M) \sum_{P} \operatorname{sgn}(D, M \Delta P) x^{w(P)}=s(D, M) \mathcal{P}(D, M) .
$$

In the construction of section 3 we rely on the following definition and theorem.

Definition 5.4 Let $G$ be a graph and let $G^{\prime}$ be an even subdivision of $G$. Let $D^{\prime}$ be an orientation of $G^{\prime}$. An orientation $D$ of $G$ induced by $D^{\prime}$ is constructed as follows: for each edge e of $G$ which was changed into a path $P_{e}$ in the construction of $G^{\prime}$, orient $e$ in the direction in which an odd number of edges of $P_{e}$ is directed in $D^{\prime}$ : this is uniquely determined since $P_{e}$ has an odd length.

Let $G$ be a graph and let $w$ be a vector of weights associated with the edges of $G$. Let $G^{\prime}$ be an even subdivision of $G$ and let $w^{\prime}$ be the vector of weights associated with the edges of $G^{\prime}$ induced by $w$. Each perfect matching $P$ of $G$ gives naturally rise to perfect matching $P^{\prime}$ of $G^{\prime}$ such that $x^{w(P)}=x^{w^{\prime}\left(P^{\prime}\right)}$.

Observe that $\operatorname{sgn}(D, P \Delta Q)=\operatorname{sgn}\left(D^{\prime}, P^{\prime} \Delta Q^{\prime}\right)$ for each pair of perfect matchings $P, Q$ of $G$. Hence the following theorem follows from Theorem 5.3.

Theorem 5.5 Let $G$ be a graph and let $w$ be a vector of weights associated with the edges of $G$. Let $G^{\prime}$ be an even subdivision of $G$ and let $w^{\prime}$ be a vector of weights associated with the edges of $G^{\prime}$ induced by $w$. Let $D^{\prime}$ be an orientation of $G^{\prime}$ and let $D$ be the orientation of $G$ induced by $D^{\prime}$. Let $M$ be an arbitrary perfect matching of $G$. Then $s(D, M) s\left(D^{\prime}, M^{\prime}\right) \operatorname{Pf}\left(A\left(D^{\prime}\right)\right)=\operatorname{Pf}(A(D))$.

An embedding of a graph on a surface is defined in a natural way: the vertices are embedded as points, and each edge is embedded as a continuous non-self-intersecting curve connecting the embedding of its end vertices. The interiors of the embedding of the edges are pairwise disjoint and the interiors of the curves embedding edges do not contain points embedding vertices.

A graph is called planar if it may be embedded on the plane. A plane graph is a planar graph together with its planar embedding. The embedding of a plane graph partitions 
the plane into connected regions called faces. The (unique) unbounded face is called outer face and the bounded faces are called inner faces.

Plane graphs with some regularities are sometimes called 2-dimensional lattices.

Let $G$ be a plane graph. A subgraph of $G$ consisting of the vertices and the edges embedded on the boundary of a face will also be called a face. If a plane graph is 2connected then each face is a cycle.

The genus $g$ of a graph $G$ is that of the orientable surface $\mathcal{S} \subset \mathbb{R}^{3}$ of minimal genus on which $G$ may be embedded. 